\title{
Association between physical and mental health-related quality of life and adverse outcomes; a retrospective cohort study of 5,272 Scottish adults
}

\author{
Zia Ul-Haq ${ }^{1,2}$, Daniel F Mackay ${ }^{1}$ and Jill P Pell ${ }^{1 *}$
}

\begin{abstract}
Background: Health-related quality of life (HRQoL) is associated with adverse outcomes in disease-specific populations. This study examines whether it is also independent predictor of incident cancer, coronary heart disease (CHD) and mortality in the general population.

Methods: The records of adult participants in the Scottish Health Survey 2003 were linked with hospital admissions, cancer registrations and death certificates. Cox proportional hazard models were used to explore the associations between quintiles of physical and mental component summary score (PCS and MCS respectively) of the SF-12 and adverse outcomes. Higher quintiles of both PCS and MCS indicate better health status.

Results: Among the 5,272 study participants, the mean PCS score was 49 (standard deviation (SD) 10.3). Participants were followed-up for a mean of 7.6 years. On survival analysis the lowest quintile of PCS was a strong predictor of all-cause death (hazard ratio (HR) 2.81, 95\% Cl 1.76, 4.49), incident cancer (HR 1.63, 95\% Cl 1.10, 2.42), and CHD events (HR 1.99, 95\% Cl 1.00, 3.96), compared to the highest quintile. This association was independent of adiposity and other confounders. The mean MCS score 52 (SD 8.8). MCS quintile was not associated with incident cancer and CHD, and the association between MCS and all-cause death (HR 1.33,95\% Cl 1.01, 1.75) became non-significant after adjustment for adiposity.
\end{abstract}

Conclusion: Physical HRQOL is a significant predictor of a range of adverse outcomes, even after adjustment for adiposity and other confounders. This study highlights the importance of perceived health in the general population.

Keywords: Health-related quality of life, HRQoL, Scottish health survey, Mortality, Adverse outcomes

\section{Background}

Studies have shown that overall health-related quality of life (HRQoL) is associated with adverse outcomes, such as hospitalisation and death, in several disease-specific populations including: patients receiving haemodialysis [1], and patients with diabetes [2], pulmonary diseases [3], coronary heart disease (CHD) [4], stroke [5], cancer [6], arthritis [7], and liver disease [8]. However, results have conflicted in relation to the associations with the physical and mental components of HRQoL. Some studies

\footnotetext{
* Correspondence: jill.pell@glasgow.ac.uk

'Institute of Health \& Wellbeing, University of Glasgow, 1 Lilybank Gardens, Glasgow G12 8RZ, UK

Full list of author information is available at the end of the article
}

have shown that physical HRQoL is significantly associated with adverse outcomes [2], but others have reported no association [3]. Similar contradictory findings have been reported for mental HRQoL $[9,10]$. There is a general paucity of studies that have examined the associations between overall, physical or mental HRQoL and adverse outcomes in the general population [11].

SF-12 is a validated and widely used tool for measuring the generic HRQoL [12]. It is a shorter version of SF-36 and takes only one third of the time to complete the SF-36 and is, therefore, used in many large surveys [13]. The 12 questions of SF-12 are combined to form summary scores for physical and mental HRQoL, called physical component summary (PCS) and mental component summary 
(MCS). These summary scores are closely correlated with those produced using the SF-36 [14].

In a recent cross-sectional study using UK Biobank participants, we demonstrated that both high and low levels of adiposity were associated with poor self-reported health [15]. This association persisted after adjustment for potential confounders and was consistent across a number of anthropometric measurements including: body mass index (BMI), waist circumference, waist to hip ratio and body fat percentage. In a retrospective cohort study of 20,000 Scottish adults with 17 years follow-up, we found that poor self-reported health (SRH) at baseline was an independent predictor of all-cause death, incident cancer, psychiatric hospitalisations and CHD events [16]. In contrast, there was no independent association between poor mental health (measured by GHQ-12) and these adverse outcomes.

There is an ongoing debate that if a single question such as, SRH is available and is consistently reported to be a reliable measure then why to use a lengthy and multiple item questionnaires such as SF-36 and SF-12. However, health status measured by SRH, GHQ-12 and different measures of HRQoL are not identical $[17,18]$. The SRH has clear advantage of reducing burden on respondents, particularly when the researchers are only interested for a broader view of overall health rather than a detail assessment. Nonetheless, SRH is a simple and reliable measure but it is at the cost of a detailed assessment on the individual domains of subjective well-being [19]. A multi-item measure, such as SF-36 and SF-12 offer more precise and complete multi-dimensional information of the individual's perception of their own health. Therefore, several indicators exist but they are not the same and should not be used interchangeably [18]. The focus of this study is the use of HRQoL as an indicator of future health outcomes.

Adiposity is significantly associated with reduced overall health-related quality of life (HRQoL), even in the absence of medical comorbidity [20]. Two recent meta-analyses showed a significant dose-response relationship between adiposity and poor physical HRQoL, whereas mental HRQoL was only reduced in the morbidly obese [21,22]. Higher BMI was also associated with many adverse outcomes, including incidence of CHD and cancer, and all-cause mortality [23,24]. Adiposity can act as a mediator in the association between HRQoL and adverse outcomes.

In this study we investigate whether physical and mental HRQoL (derived from the SF-12) were independent predictors of incident cancer, CHD events, and all-cause deaths, and whether the associations varied by sex and BMI among a large representative sample from the Scotland adult population, after adjusting for potential confounding factors including: demographic and life-style factors, socio-economic status, hypertension, diabetes and adiposity.

\section{Methods}

\section{Data sources}

We used an extract of data from the Scottish Health Survey (SHS) 2003. (http://www.scotland.gov.uk/Topics/ Statistics/Browse/Health/scottish-health-survey). Unlike, the earlier two SHS's, which were conducted in 1995 and 1998, the SHS 2003 had no age limitation and it is the only SHS which included the SF-12 questionnaire to measure the physical and mental HRQoL. The details of the SHS have been described previously $[20,25]$. In brief, participants were interviewed face to face by trained staff who collected information on demographics (including age and sex), socio-economic status (including area of residence and level of education) and lifestyle behaviours (including smoking habits and alcohol consumption) as well as completing the SF-12 questionnaire. The data collectors also measured the weight and height of study participants. In a follow-up visit, a qualified nurse measured blood pressure. The overall response rate was around 60\%. Furthermore, over $90 \%$ of SHS participants consented to passive follow-up via record linkage to the Scotland-wide routine administrative databases held by the Information Services Division including: admissions to acute hospital (Scottish Morbidity Record SMR01), cancer registrations (Scottish Morbidity Record SMR06) and death certificates [26]. The SMR data undergo regular quality assurance checks and have been shown to be $99 \%$ complete and $94 \%$ accurate [27]. The linkage provided follow-up data up to a censor date of 31 December 2011.

\section{Inclusion criteria and definitions}

SF-12 questionnaires were completed by SHS participants aged $\geq 20$ years. Therefore our study was restricted to this age-group. Participants with a history of cancer or CHD at the time of the baseline interview were excluded from the study. Study participants were categorized into $20-44,45-64$ and $\geq 65$ years of age. BMI was categorized, using standardized cut-off points, into underweight $\left(<18.5 \mathrm{~kg} / \mathrm{m}^{2}\right)$, normal weight $\left(18.5-24.9 \mathrm{~kg} / \mathrm{m}^{2}\right)$, overweight $\left(25.0-29.9 \mathrm{~kg} / \mathrm{m}^{2}\right)$, and obese $\left(\geq 30 \mathrm{~kg} / \mathrm{m}^{2}\right)$. Obese was further categorized into class I $\left(30.0-34.9 \mathrm{~kg} / \mathrm{m}^{2}\right)$, class II $\left(35.0-39.9 \mathrm{~kg} / \mathrm{m}^{2}\right)$ and class III obese $\left(\geq 40 \mathrm{~kg} / \mathrm{m}^{2}\right)$. The level of education was treated as four categories from level 1 (less than O level grade C) to level 4 (degree level or above). The Scottish Index of Multiple deprivation (SIMD) was used as the measure of socio-economic status. SIMD is a validated and widely used area-based measure of multiple deprivations and is derived from participants' postcodes of residence. SIMD is calculated using 31 indicators across 6 domains: income, employment, housing, health, education, skills and training, and area based access to the services (http://www.scotland.gov.uk/ Topics/Statistics/SIMD). Self-reported smoking status was 
categorized into never, previous or current, and alcohol consumption was categorized as never, previous, within limits ( $<21$ units/week for men; $<14$ units/week for women) and excessive. Hypertension was defined as $\geq 140$ / $90 \mathrm{mmHg}$ or use of anti-hypertensive medication. Presence of diabetes was based on self-report of a physician diagnosis. PCS and MCS were calculated from the 12 responses to the SF-12 questionnaire. Higher scores indicate better physical and mental health status respectively. For the ease of interpretation, PCS and MCS score quintiles were used in the analyses. Cancer was defined using International Classification of Diseases-10 (ICD-10) codes C00-C97. CHD event was defined as death or hospitalisation due to CHD. The latter was defined as first hospitalisation using ICD-10 code I20-I25 in the primary position of diagnosis.

\section{Statistical analyses}

The characteristics of participants by quintile of PCS and MCS were analysed using chi-square tests or chi-square tests for trend for binary and ordinal data respectively. Separate Cox proportional hazard models were used to examine the associations between PCS and MCS quintile and three outcomes: all-cause deaths, cancer registrations, and CHD events (hospitalisations or death). The highest quintile (best HRQoL) was used as the referent category. The models were first adjusted for age only (model 1), followed by further adjustment for sex, SIMD, education level, smoking status, alcohol consumption, medical comorbidity (hypertension and diabetes) (model 2), and finally BMI was added as a covariate (model 3). Global test was used to check the proportional-hazards assumption of our survival models. We were interested in exploring whether sex, BMI or lifestyle behaviours could modify the relationship between HRQoL and outcomes, and thus influence the assessment of health outcomes and mortality by using HRQoL. Therefore we tested for statistical interactions between HRQoL summary scores and sex, BMI and lifestyle behaviours. All statistical analyses were performed using Stata version 12.1 (StataCorp, College Station, Texas). Statistical significance was defined as $\mathrm{p}<0.05$.

\section{Ethical approval}

Scottish Health Survey participants consented to passive follow-up via record linkage to the Scotland-wide routine administrative databases held by the Information Services Division including: admissions to acute hospital. The authors used secondary analyses of health survey data using an anonymised data extract.

\section{Results}

Of the 5,272 participants, 2,889 (54.8\%) were women, 1,392 (26.4\%) were current smokers, 1,096 (20.8\%) consumed excessive amounts of alcohol and 1,316 (25\%) had either hypertension, diabetes or both (Additional file 1). Their mean age at recruitment was 50 years (SD 16 years). The mean BMI was $27.5 \mathrm{~kg} / \mathrm{m}^{2}$ (SD $5.1 \mathrm{~kg} / \mathrm{m}^{2}$ ); 59 (1.1\%) were underweight, 1,689 (32\%) normal-weight, 2,152 (40.8\%) overweight, and 1,372 (26\%) obese. Of the obese, 940 (17.8\%) were class I, 297 (5.6\%) were class II, and 135 (2.6\%) were class III obese (Table 1). Participants were followed-up for a maximum of 8 years (mean 7.6 years), providing a total of 40,067.2 person years of follow-up. Incident events included 391 (7.4\%) all-cause deaths, 368 (7.0\%) cancer registrations, and 134 (2.5\%) CHD hospitalisations or deaths.

Overall, the mean PCS score was 49 (SD 10.3). The lowest quintile equated to $<42$ and the highest to $>56$. Compared to the participants who were in the highest quintile of PCS (better physical HRQoL), those in the lowest quintile were older and more likely to be obese, male, socio-economically deprived, smoke and have hypertension or diabetes, but were less likely to consume excessive amounts of alcohol or have a higher degree (Table 1). Overall, the mean MCS score was 52 (SD 8.8). The lowest quintile equated to $<47$ and the highest quintile to $>58$. There was a U-shaped relationship between BMI, level of education and socio-economic deprivation and higher MCS score (Table 1). Compared to the participants who were in the highest quintile of MCS (better mental HRQoL), those in the lowest quintile had similar proportions of obese, hypertension or diabetes, not socio-economically deprived, and educated (Table 1). Unlike, PCS there was no dose-response relationship between age, BMI, deprivation and hypertension or diabetes and MCS.

The Cox-proportional hazard models revealed that there were inverse dose-response relationships between baseline PCS and all-cause deaths, cancer registrations, and CHD events (Figure 1). Compared to those in the highest quintile (better physical HRQoL), participants in bottom two quintiles were significantly more likely to experience all-cause death, cancer registration and CHD events when adjusted for age only (Table 2). Further adjustment for sex, socioeconomic status, education level, smoking status, alcohol consumption, hypertension and diabetes attenuated the associations but the participants in the lowest quintile of PCS remained at significantly higher risk of adverse outcomes, compared to the highest quintile of PCS. When BMI was added to the model the lowest quintile of PCS remained a significant predictor of all-cause death (HR 2.64, 95\% CI 1.76, 4.49), incident cancer (HR 1.63, 95\% CI 1.10, 2.42), and CHD events (HR 1.99, 95\% CI 1.00, 3.96), compared to the participants in the highest PCS quintile (PCS score $>56$ ) (Table 2). There was no significant interaction between PCS quintile and either sex $(p=0.968)$, BMI $(p=0.059)$, 
Table 1 Characteristics of the participants by physical and mental component summary score quintiles of the SF-12

\begin{tabular}{|c|c|c|c|c|c|c|c|c|c|c|c|c|}
\hline & \multicolumn{6}{|c|}{ Physical component quintile (score) } & \multicolumn{6}{|c|}{ Mental component quintile (score) } \\
\hline & $1(<42)$ & $2(42-50.9)$ & $3(51-54.9)$ & $4(55-56)$ & $5(>56)$ & & $1(<47)$ & $2(47-52.9)$ & $3(53-55.9)$ & $4(56-58)$ & $5(>58)$ & \\
\hline & $N=1,055$ & $N=1,054$ & $N=1,057$ & $N=1,068$ & $N=1,038$ & & $N=1,055$ & $N=1,054$ & $N=1,057$ & $N=1,068$ & $N=1,038$ & \\
\hline & N (\%) & N (\%) & $\mathrm{N}(\%)$ & N (\%) & N (\%) & $P$ value & N (\%) & N (\%) & N (\%) & N (\%) & N (\%) & $P$ value \\
\hline \multicolumn{13}{|l|}{ Body Mass Index } \\
\hline Underweight & $24(2.3)$ & $4(0.38)$ & $9(0.85)$ & $11(1.0)$ & $11(1.1)$ & $<0.001$ & $21(2.0)$ & $4(0.4)$ & $15(1.4)$ & $11(1.0)$ & $8(0.8)$ & 0.045 \\
\hline Normal-weight & $251(23.8)$ & $287(27.2)$ & $330(31.2)$ & $358(33.5)$ & $463(44.6)$ & & $338(31.7)$ & $365(34.0)$ & $379(36.0)$ & $358(32.6)$ & $249(25.4)$ & \\
\hline Overweight & $369(35.0)$ & $416(39.5)$ & $445(42.1)$ & $496(46.4)$ & $426(41.0)$ & & 391 (36.7) & $435(40.5)$ & 441 (41.9) & $484(44.0)$ & 401 (40.9) & \\
\hline Obese & $411(39.0)$ & $347(32.9)$ & $273(25.8)$ & $203(19.0)$ & $138(13.3)$ & & 315 (29.6) & $271(25.2)$ & 217 (20.6) & $247(22.5)$ & $322(32.9)$ & \\
\hline Class I & $261(24.7)$ & $222(21.1)$ & $202(19.1)$ & $148(13.9)$ & $107(10.3)$ & & $184(17.3)$ & $190(17.7)$ & $162(15.4)$ & $171(15.6)$ & $233(23.8)$ & \\
\hline Class II & $95(9.0)$ & $95(9.0)$ & $48(4.5)$ & $34(3.2)$ & $25(2.4)$ & & $81(7.6)$ & $54(5.0)$ & $39(3.7)$ & $56(5.1)$ & $67(6.8)$ & \\
\hline Class III & $55(5.2)$ & $30(2.9)$ & $23(2.2)$ & $21(2.0)$ & $6(0.6)$ & & $50(4.7)$ & $27(2.5)$ & $16(1.5)$ & $20(1.8)$ & $22(2.2)$ & \\
\hline \multicolumn{13}{|l|}{ Sex } \\
\hline Men & $463(43.9)$ & $476(45.2)$ & $508(48.1)$ & $508(47.6)$ & $428(41.2)$ & $<0.001$ & 419 (39.3) & $448(41.7)$ & $488(46.4)$ & $532(48.4)$ & $496(50.6)$ & $<0.001$ \\
\hline Women & $592(56.1)$ & $578(54.8)$ & $549(51.9)$ & $560(52.4)$ & $610(58.8)$ & & $646(60.7)$ & $627(58.3)$ & $564(53.6)$ & $568(51.6)$ & $484(49.4)$ & \\
\hline \multicolumn{13}{|l|}{ Age (years) } \\
\hline $20-44$ & $201(19.1)$ & $404(38.3)$ & $507(48.0)$ & $527(49.3)$ & $573(55.2)$ & $<0.001$ & $473(44.4)$ & $508(47.3)$ & $518(49.2)$ & $484(44.0)$ & $229(23.4)$ & $<0.001$ \\
\hline $45-64$ & $419(39.7)$ & $400(38.0)$ & 395 (37.4) & $403(37.7)$ & $389(37.5)$ & & 409 (38.4) & $422(39.3)$ & $386(36.7)$ & $416(37.8)$ & $373(38.1)$ & \\
\hline$\geq 65$ & $435(41.2)$ & $250(23.7)$ & $155(14.7)$ & 138 (12.9) & $76(7.3)$ & & $183(17.2)$ & 145 (13.5) & $148(14.1)$ & $200(18.2)$ & $378(38.6)$ & \\
\hline \multicolumn{13}{|l|}{ SIMD } \\
\hline 1 (most deprived) & $267(25.3)$ & $197(18.7)$ & $153(14.5)$ & $157(14.5)$ & $105(10.1)$ & $<0.001$ & $246(23.1)$ & $182(16.9)$ & $154(14.6)$ & $145(13.2)$ & $152(15.5)$ & $<0.001$ \\
\hline 2 & $247(23.4)$ & $197(18.7)$ & $204(19.3)$ & $179(16.8)$ & $168(16.8)$ & & $233(21.9)$ & $187(17.4)$ & $182(17.3)$ & $196(17.8)$ & $197(20.1)$ & \\
\hline 3 & $238(22.6)$ & $254(24.1)$ & $257(24.3)$ & $220(20.6)$ & $253(24.4)$ & & $253(23.8)$ & $235(21.7)$ & $234(22.2)$ & $253(23.0)$ & $247(25.2)$ & \\
\hline 4 & 189 (17.9) & 209 (19.8) & $230(21.8)$ & $248(23.2)$ & $252(24.3)$ & & 198 (18.6) & $240(22.3)$ & 234 (22.2) & $223(20.3)$ & $233(23.8)$ & \\
\hline 5 (least deprived) & $114(10.8)$ & $197(18.7)$ & $213(20.2)$ & $264(24.7)$ & $260(25.1)$ & & 135 (12.7) & $231(21.5)$ & $248(23.6)$ & $283(25.7)$ & $151(15.4)$ & \\
\hline \multicolumn{13}{|l|}{ Education $^{\mathrm{a}}$} \\
\hline Level 1 & $139(13.2)$ & $168(15.9)$ & $170(16.1)$ & $187(17.5)$ & $169(16.3)$ & $<0.001$ & $170(15.7)$ & 185 (17.2) & 175 (16.6) & $166(15.1)$ & $137(14.0)$ & 0.015 \\
\hline Level 2 & 143 (13.6) & $163(15.5)$ & 193 (18.3) & $198(18.5)$ & $200(19.3)$ & & $167(15.7)$ & $175(16.3)$ & 211 (20.1) & $209(19.0)$ & $135(13.8)$ & \\
\hline Level 2 & $32(3.0)$ & 77 (7.31) & $78(7.4)$ & $92(8.6)$ & $93(9.0)$ & & $167(7.8)$ & $175(6.1)$ & $211(7.5)$ & 209 (8.3) & $135(5.5)$ & \\
\hline Level 4 & $151(14.3)$ & $210(19.9)$ & $290(27.4)$ & $314(29.4)$ & $400(38.5)$ & & $224(21.0)$ & 321 (29.9) & $326(31.0)$ & $289(26.3)$ & 205 (20.9) & \\
\hline None of these & $590(55.9)$ & $436(41.4)$ & $326(30.8)$ & $277(25.9)$ & $176(17.0)$ & & $421(39.5)$ & $329(30.6)$ & $261(24.8)$ & $345(31.4)$ & 449 (45.8) & \\
\hline \multicolumn{13}{|l|}{ Smoking status } \\
\hline Never & $361(34.2)$ & $441(41.9)$ & $447(42.3)$ & $526(49.3)$ & $541(52.1)$ & $<0.001$ & 390 (36.6) & $465(43.3)$ & $490(46.6)$ & $536(48.7)$ & $435(44.4)$ & $<0.001$ \\
\hline
\end{tabular}


Table 1 Characteristics of the participants by physical and mental component summary score quintiles of the SF-12 (Continued)

\begin{tabular}{|c|c|c|c|c|c|c|c|c|c|c|c|c|}
\hline Previous & $387(36.7)$ & $317(30.1)$ & $296(28.0)$ & $306(28.7)$ & $258(24.9)$ & & $268(25.2)$ & $311(28.9)$ & $318(30.2)$ & $319(29.0)$ & $348(35.5)$ & \\
\hline Current & $307(29.1)$ & $296(28.1)$ & $314(29.7)$ & $236(22.1)$ & $239(23.0)$ & & $407(38.2)$ & $299(27.8)$ & $244(23.2)$ & $245(22.3)$ & $197(20.1)$ & \\
\hline \multicolumn{13}{|c|}{ Alcohol consumption } \\
\hline Never & $92(8.7)$ & $53(5.03)$ & $36(3.4)$ & $39(3.7)$ & $32(3.1)$ & $<0.001$ & $53(5.0)$ & $41(3.8)$ & $34(3.2)$ & $42(3.8)$ & $82(8.4)$ & 0.016 \\
\hline Previous & $99(9.4)$ & $39(3.7)$ & $36(3.4)$ & $26(2.4)$ & $25(2.4)$ & & $75(7.0)$ & $39(3.6)$ & $35(3.3)$ & $37(3.4)$ & $39(4.0)$ & \\
\hline Within limit & $704(66.7)$ & $739(70.1)$ & $733(69.4)$ & $802(75.1)$ & $721(69.5)$ & & $687(64.5)$ & $774(72.0)$ & $748(71.1)$ & $812(73.8)$ & $678(69.2)$ & \\
\hline Excessive & $160(15.2)$ & $223(21.2)$ & $252(23.8)$ & $201(18.8)$ & $260(25.1)$ & & $250(23.5)$ & $221(20.6)$ & $235(22.3)$ & $209(19.0)$ & $181(18.5)$ & \\
\hline \multicolumn{13}{|c|}{ Medical comorbidity } \\
\hline No & $576(54.6)$ & $725(68.8)$ & $829(78.4)$ & $886(83.0)$ & $940(90.6)$ & $<0.001$ & $734(68.9)$ & $836(77.8)$ & $859(81.7)$ & $879(79.9)$ & $648(66.1)$ & $<0.001$ \\
\hline Yes & $479(45.4)$ & $329(31.2)$ & $228(21.6)$ & $182(17.0)$ & $98(9.4)$ & & $331(31.1)$ & $239(22.2)$ & $193(18.4)$ & $221(20.1)$ & $332(33.9)$ & \\
\hline
\end{tabular}

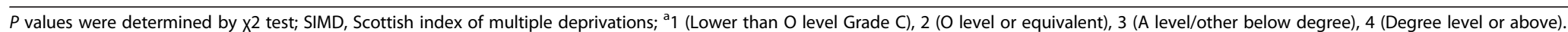




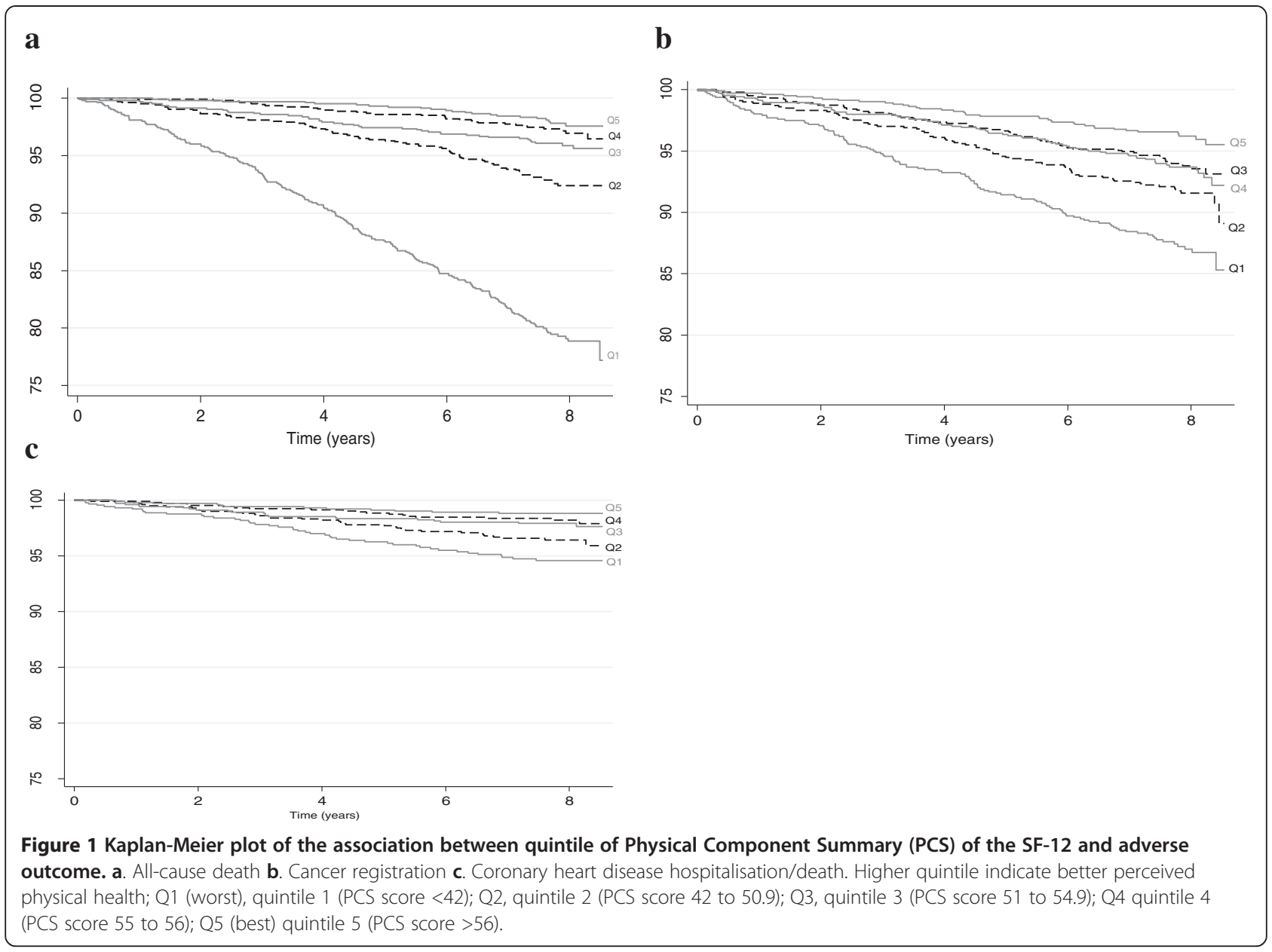

smoking $(\mathrm{p}=0.069)$ or alcohol consumption $(\mathrm{p}=0.328)$ in relation to any of the adverse outcomes.

There were inverse dose response relationships between the two lowest quintiles of MCS and all-cause death, but not with CHD events or cancer registration. Being in the lowest quintile of MCS was a significant predictor of all-cause death after adjustment for age (Table 3). When further adjusted for sex, socioeconomic status, education level, smoking status, alcohol consumption, hypertension and diabetes the hazard ratios were attenuated but remained statistically significant (HR 1.33, 95\% CI 1.01, 1.75). When also adjusted for BMI, the association became statistically non-significant. There was no significant interaction between MCS and either sex $(p=0.062)$ or BMI $(\mathrm{p}=0.767)$, or alcohol consumption $(\mathrm{p}=0.367)$ in relation to any of the adverse outcomes.

\section{Discussion}

Physical HRQoL was found to be a strong predictor of incident cancer, CHD events and all-cause mortality on follow-up. The associations were independent of adiposity and other potential confounders, and there was evidence of inverse dose-response relationships. In contrast, poor mental HRQoL was only a significant predictor of all-cause death and that was explained by adiposity. There were no statistically significant differences in the associations between men and women or by level of adiposity.

The majority of previous studies have focused on the association between HRQoL and mortality in diseases-specific populations and they have produced conflicting results. For example, in patients undergoing haemodialysis some studies have reported that both PCS and MCS were strong predictors of mortality [1]. Some reported that MCS was a significant predictor of mortality, but not PCS [10]. Others reported the reverse findings with PCS being a significant predictor of mortality, but not MCS [9]. In patients with pulmonary fibrosis, HRQoL did not predict death [3]. PCS was associated with higher mortality in diabetic patients, but not MCS [2]. In a study of patients with heart failure, MCS predicted mortality, but PCS did not [4]. Others have reported that both MCS and PCS were associated with higher mortality in atherosclerotic patients [5]. Similarly, only PCS was strongly associated with mortality in patients with rheumatoid arthritis [7], but both PCS and MCS were 
Table 2 Cox regression models of the association between quintiles of physical component summary score (PCS) of the SF-12 and adverse outcomes

\begin{tabular}{|c|c|c|c|c|c|c|}
\hline & \multicolumn{2}{|c|}{ Model 1} & \multicolumn{2}{|c|}{ Model 2} & \multicolumn{2}{|c|}{ Model 3} \\
\hline & HR (95\% Cl) & $P$ value & HR (95\% Cl) & $P$ value & HR $(95 \% \mathrm{Cl})$ & $P$ value \\
\hline \multicolumn{7}{|c|}{ All-cause death } \\
\hline \multicolumn{7}{|l|}{ PCS quintile } \\
\hline 1 (worst) & $4.23(2.70,6.63)$ & $<0.001$ & $2.64(1.66,4.20)$ & $<0.001$ & $2.81(1.76,4.49)$ & $<0.001$ \\
\hline 2 & $1.97(1.22,3.19)$ & 0.005 & $1.44(0.88,2.34)$ & 0.146 & $1.55(0.95,2.54)$ & 0.078 \\
\hline 3 & $1.45(0.86,2.42)$ & 0.160 & $1.09(0.65,1.84)$ & 0.747 & $1.15(0.68,1.94)$ & 0.599 \\
\hline 4 & $1.06(0.61,1.84)$ & 0.826 & $0.90(0.52,1.57)$ & 0.718 & $0.95(0.55,1.66)$ & 0.869 \\
\hline 5 (best) & 1.00 & - & 1.00 & - & 1.00 & - \\
\hline \multicolumn{7}{|c|}{ Cancer registration } \\
\hline \multicolumn{7}{|l|}{ PCS quintile } \\
\hline 1 (worst) & $1.87(1.29,2.71)$ & 0.001 & $1.60(1.08,2.37)$ & 0.018 & $1.63(1.10,2.42)$ & 0.015 \\
\hline 2 & $1.58(1.07,2.31)$ & 0.020 & $1.45(0.98,2.14)$ & 0.067 & $1.47(0.99,2.19)$ & 0.056 \\
\hline 3 & $1.39(0.93,2.07)$ & 0.110 & $1.29(0.86,1.93)$ & 0.223 & $1.30(0.86,1.95)$ & 0.212 \\
\hline 4 & $1.50(1.01,2.23)$ & 0.043 & $1.46(0.98,2.18)$ & 0.062 & $1.45(0.98,2.17)$ & 0.066 \\
\hline 5 (best) & 1.00 & - & 1.00 & - & 1.00 & - \\
\hline \multicolumn{7}{|c|}{ Coronary heart disease hospitalisation/death } \\
\hline \multicolumn{7}{|l|}{ PCS quintile } \\
\hline 1 (worst) & $2.57(1.35,4.93)$ & 0.004 & $1.99(1.01,3.93)$ & 0.047 & $1.99(1.00,3.96)$ & 0.049 \\
\hline 2 & $2.23(1.15,4.30)$ & 0.017 & $1.82(0.93,3.59)$ & 0.082 & $1.81(0.92,3.58)$ & 0.087 \\
\hline 3 & $1.61(0.79,3.25)$ & 0.187 & $1.36(0.67,2.78)$ & 0.398 & $1.36(0.66,2.78)$ & 0.402 \\
\hline 4 & $1.36(0.66,2.80)$ & 0.405 & $1.19(0.57,2.47)$ & 0.646 & $1.19(0.57,2.48)$ & 0.640 \\
\hline 5 (best) & 1.00 & - & 1.00 & - & 1.00 & - \\
\hline
\end{tabular}

Higher quintile indicate better physical health status; PCS, physical component summary quintile (score): 1 (<42), 2 (42 to 50.9$), 3(51$ to 54.9$), 4(55$ to 56$), 5$ ( $>56)$; $\mathrm{HR}$, hazard ratio; Cl, Confidence interval; Model 1 adjusted for age; Model 2 adjusted for age, sex, SIMD, education level, smoking status, alcohol consumption, hypertension and diabetes; Model 3 adjusted for same covariates as Model 2 plus body mass index.

in patients with liver cirrhosis [8]. One recent study reported that HRQoL was the only psychosocial predictor of survival in cancer patients [6].

Very few studies have explored the association between HRQoL and adverse outcomes in the general population. In a recent German study, 4,259 participants, aged 20-79 years, completed the SF-12 at baseline and suffered 456 deaths over a mean of 9.7 years follow-up [11]. The Cox-proportional hazard models revealed that the lowest quartile of PCS was an independent predictor of mortality (fully adjusted HR 1.64, 95\% CI 1.19, 2.27), compared to the highest quartile. In contrast, MCS was not a significant predictor of mortality (HR 0.97, 95\% CI $0.74,1.28$ ). Other studies conducted in general population have been few in number and have focused mainly on people aged 60 years or more. A longitudinal study was conducted in Taiwan, in which 4,424 individuals, aged 65 years and older were followed over three years and 221 deaths recorded [28]. A 10-point decrease in both PCS and MCS scores was associated with higher mortality; relative risk (RR) 1.60, 95\% CI 1.39, 1.83, and RR 1.16, $95 \%$ CI 1.01, 1.34, respectively. In a US study 2,166 participants, aged 65 years or older, completed SF-12 questionnaires at baseline and were passively followed-up over 28 months using data from their insurance records [29]. Participants in the lowest quartile of PCS had a higher risk of both all-cause deaths (HR 5.99, 95\% CI 1.90, 18.95) and hospitalisation (HR 2.64, p < 0.001) than those in the highest quartile. Those in the lowest quartile for MCS were also at higher risk of death (HR 2.30, 95\% CI 1.64, 3.22) and hospitalisation (HR 1.40, $\mathrm{p}<0.001$ ). A Spanish study followed 2,343 participants, aged 60 years and above for six years and recorded 212 deaths [30]. A five-point decrease in baseline PCS score was found to be a significant predictor of mortality (HR 1.28, $95 \%$ CI 1.17, 1.40) but this was not true for MCS (HR 1.05, 95\% CI 0.97, 1.13). Our model 2 results are consistent with these previous studies.

Recently, in a larger study of the Scottish adult population, with follow-up of 17 years, we demonstrated that after full adjustment, poor baseline self-reported health was an independent predictor of all-cause death (HR 2.48 95\% CI 2.16, 2.85), incident cancer (HR 1.32, 95\% CI 1.09, 1.58), and CHD events (HR 2.26, 95\% CI 1.79, 2.84) [16]. 
Table 3 Cox regression models of the association between quintiles of mental component summary score (MCS) of the SF-12 and adverse outcomes

\begin{tabular}{|c|c|c|c|c|c|c|}
\hline & \multicolumn{2}{|c|}{ Model 1} & \multicolumn{2}{|c|}{ Model 2} & \multicolumn{2}{|c|}{ Model 3} \\
\hline & HR (95\% Cl) & $P$ value & HR (95\% Cl) & $P$ value & HR (95\% Cl) & $P$ value \\
\hline \multicolumn{7}{|c|}{ All-cause death } \\
\hline \multicolumn{7}{|l|}{ MCS quintile } \\
\hline 1 (worst) & $1.61(1.24,2.10)$ & $<0.001$ & $1.33(1.01,1.75)$ & 0.041 & $1.25(0.95,1.65)$ & 0.117 \\
\hline 2 & $1.04(0.76,1.41)$ & 0.824 & $1.00(0.73,1.37)$ & 0.991 & $1.00(0.73,1.37)$ & 0.998 \\
\hline 3 & $0.83(0.59,1.15)$ & 0.259 & $0.92(0.66,1.28)$ & 0.603 & $0.88(0.63,1.23)$ & 0.463 \\
\hline 4 & $0.66(0.47,0.91)$ & 0.012 & $0.77(0.56,1.08)$ & 0.128 & $0.76(0.55,1.06)$ & 0.104 \\
\hline 5 (best) & 1.00 & - & 1.00 & - & 1.00 & - \\
\hline \multicolumn{7}{|c|}{ Cancer registration } \\
\hline \multicolumn{7}{|l|}{ MCS quintile } \\
\hline 1 (worst) & $0.81(0.59,1.10)$ & 0.171 & $0.75(0.55,1.03)$ & 0.077 & $0.75(0.55,1.03)$ & 0.079 \\
\hline 2 & $0.75(0.55,1.03)$ & 0.077 & $0.75(0.54,1.03)$ & 0.075 & $0.74(0.54,1.03)$ & 0.071 \\
\hline 3 & $0.70(0.51,0.97)$ & 0.033 & $0.73(0.52,1.01)$ & 0.055 & $0.72(0.52,1.01)$ & 0.054 \\
\hline 4 & $0.78(0.58,1.06)$ & 0.111 & $0.83(0.61,1.12)$ & 0.215 & $0.82(0.61,1.11)$ & 0.206 \\
\hline 5 (best) & 1.00 & - & 1.00 & - & 1.00 & - \\
\hline \multicolumn{7}{|c|}{ Coronary heart disease hospitalisation/death } \\
\hline \multicolumn{7}{|l|}{ MCS quintile } \\
\hline 1 (worst) & $0.91(0.56,1.48)$ & 0.715 & $0.84(0.51,1.37)$ & 0.480 & $0.84(0.51,1.38)$ & 0.499 \\
\hline 2 & $0.60(0.35,1.05)$ & 0.072 & $0.61(0.35,1.06)$ & 0.077 & $0.61(0.35,1.06)$ & 0.082 \\
\hline 3 & $0.55(0.31,0.97)$ & 0.039 & $0.59(0.33,1.05)$ & 0.071 & $0.59(0.33,1.05)$ & 0.073 \\
\hline 4 & $0.75(0.46,1.22)$ & 0.252 & $0.79(0.48,1.29)$ & 0.352 & $0.80(0.49,1.30)$ & 0.363 \\
\hline 5 (best) & 1.00 & - & 1.00 & - & 1.00 & - \\
\hline
\end{tabular}

Higher quintile indicate better mental health status; MCS, mental component summary quintile (score): 1 (<47), 2 (47 to 52.9$), 3(53$ to 55.9$), 4(56$ to 58$), 5$ (>58); $\mathrm{HR}$, hazard ratio; Cl, Confidence interval; Model 1 adjusted for age; Model 2 adjusted for age, sex, SIMD, education level, smoking status, alcohol consumption, hypertension and diabetes; Model 3 adjusted for same covariates as Model 2 plus body mass index.

In contrast, after full adjustment, mental health (measured by GHQ-12) was not a significant predictor of these adverse outcomes. Similarly, in this study we showed independent associations between lowest quintile of baseline PCS (poor physical HRQoL) and all-cause death (HR 2.81, 95\% CI 1.76, 4.49), incident cancer (HR 1.63, 95\% CI 1.10, 2.42), and CHD events (HR 1.99, $95 \%$ CI 1.00, 3.96). In contrast, MCS was not associated with these adverse outcomes after adjustment for potential confounders.

We did not explore the underlying mechanism by which HRQoL may impact on morbidity and mortality. Self-perceived health is more inclusive and provides additional information by incorporating both objectively measured and subjective assumptions of health risk [31]. HRQoL may identify accurate health status by covering those aspects of health which are difficult to capture by objective measurements such as subclinical disease, help-seeking behaviour and health system [32]. Better HRQoL may reflect an individual's attitude towards improving health, and thus adopting preventive measures. In contrast, the low HRQoL may result in neglecting of the primary or secondary prevention such as healthy diet, physical activity, screening and taking regular medication for existing medical conditions which may eventually result in early death or disease incidence [33]. MCS was not associated with health outcomes. The exact mechanism is not known but there was a dose-response relationship between base-line BMI, level of education, hypertension, diabetes and socio-economic deprivation and low PCS. In contrast, there was a U-shaped relationship with MCS. Our current findings that comorbidity, ageing, social and obesity gradient do not exist for MCS could partly explain the fact that the participants with low MCS has lower risk of cancer incidence and CVD events, compared to the high MCS.

We have previously conducted several studies on the association between adiposity and HRQoL [15,20,25] including two meta-analyses; one in children and adolescents [21] and the other in adults [22]. Collectively, these studies revealed that adiposity had a significant positive association with poor physical HRQoL with evidence of dose response. Adiposity is also associated with many non-communicable diseases, including CHD and cancers 
[34-36]. Recently, three large-scale meta-analyses have consistently reported that obesity is a significant predictor of mortality [37-39]. In this study we have shown that poor physical HRQoL is a strong independent predictor of all-cause death, cancer incidence and CHD events. In contrast mental HRQoL is a predictor of all-cause deaths but not independently of adiposity. It is possible that mental HRQoL and adiposity lie on the same causal pathway. It is not possible to be certain of the direction of effect. Poor mental HRQoL may impact on lifestyle and, therefore, increase the risk of adiposity. Conversely, adiposity may itself predispose to poor mental HRQoL.

Commonly, population health is measured in terms of morbidity and mortality. Our results further strengthen the growing evidence that perceived health provides additional information and is predictive of future morbidity and mortality. It should be considered when undertaking both individual and community health assessments. It has been suggested that perceived health may be a stronger predictor of adverse outcomes than many objective measures of health [33].

We used data from a large representative sample of Scottish general population, and adjusted our analyses for a series of potential confounders. The "representativeness" of a health survey is generally determined by the higher number of responses which is based on the updated and correct sampling frame, study design and non-responses. The Scottish health survey has rigorous methodology and maintains the overall higher response rate of $60 \%$ or above from the eligible households. The age and sex proportion of the adult respondents is externally validated with the General Registrar Office for Scotland mid-year population estimates. The Scottish health survey used weighting to take account of the underrepresentation of the large households responses and non-response biases. It is also considered to be representative of the Scottish population in terms of the SIMD quintiles [26]. BMI and blood pressure were measured by trained individuals using standard operating procedures. The presence of diabetes was self-reported but based on physician diagnoses. The SF-12 is a validated and very widely used measure of HRQoL in the general population [12]. The Scottish Morbidity Record (SMR) has pan-Scotland coverage undergoes regular quality assurance checks [27]. Use of a cohort design enabled us to demonstrate a temporal relationship between baseline PCS and MCS and incident disease, and all-cause mortality and thereby avoid reverse causation. The advantage of retrospective cohort study includes readily access to data but it is at the cost of lack of control over the data collection. By treating PCS and MCS as ordinal data we were able to examine whether there were evidence of a dose relationship. Previous studies have also used the quintiles of PCS and MCS for the ease of interpretation $[11,40]$ but it may lead to considerable power loss and misleading results. We have used the SF-12 PCS and MCS scoring system for the SF-12 but the alternative scoring systems are also available such as RAND-12 and multidimensional item response theory (MIRT) models [17,41]. Previous studies suggested that these alternatives models may have more power to detect the true differences and can also provide more reliable and intuitive scoring, particularly when the SF-12 is used to assess the mental health status $[17,41]$.

\section{Conclusions}

Poor physical health-related quality of life is a strong predictor of all-cause death, cancer incidence and CHD events. The association is independent of adiposity and other potential confounders. This study adds to the growing evidence that perceived health is an important predictor of health risk, independent of adiposity or comorbidity, and should be considered when assessing the health of individuals and communities.

\section{Additional file}

Additional file 1: Characteristics of the participants $(n=5,272)$.

\section{Abbreviations}

HRQoL: Health-related quality of life; CHD: Coronary heart disease; SHS: Scottish Health Survey; PCS: Physical component summary; MCS: Mental component summary; HR: Hazard ratio; Cl: Confidence interval; BMI: Body mass index; NHS: National Health Service; SMR: Scottish Morbidity Record; SIMD: Scottish Index of Multiple deprivation.

\section{Competing interests}

The authors declare that they have no competing interests.

\section{Authors' contributions}

All authors contributed to conception and design. ZUH and DFM analysed the data. All authors agreed what analyses were required and interpreted the results. ZUH wrote the first draft. All authors revised the manuscript and approved the final version and takes full responsibility for the manuscript.

\section{Acknowledgment}

Dr. Zia Ul-Haq is sponsored by the Higher Education Commission, Pakistan "Development of Khyber Medical University".

\section{Financial disclosure}

No external funding received.

\section{Author details}

${ }^{1}$ Institute of Health \& Wellbeing, University of Glasgow, 1 Lilybank Gardens, Glasgow G12 8RZ, UK. Institute of Public Health \& Social Sciences (IPH \& SS), Khyber Medical University, Peshawar, KPK, Pakistan.

Received: 10 March 2014 Accepted: 30 October 2014

Published: 21 November 2014

\section{References}

1. Kalantar-Zadeh K, Kopple JD, Block G, Humphreys MH: Association among SF36 quality of life measures and nutrition, hospitalization, and mortality in hemodialysis. J Am Soc Nephrol 2001, 12:2797-2806.

2. Li CL, Chang HY, Hsu CC, Lu JF, Fang HL: Joint predictability of health related quality of life and leisure time physical activity on mortality risk in people with diabetes. BMC Public Health 2013, 13:67. 
3. Nishiyama O, Taniguchi H, Kondoh Y, Kimura T, Kataoka K, Nishimura K, Ogawa T, Watanabe F, Arizono S, Tohda Y: Health-related quality of life does not predict mortality in idiopathic pulmonary fibrosis. Sarcoidosis Vasc Diffuse Lung Dis 2012, 29:113-118.

4. Zuluaga MC, Guallar-Castillon P, Lopez-Garcia E, Banegas JR, Conde-Herrera M, Olcoz-Chiva M, Rodríguez-Pascual C, Rodriguez-Artalejo F: Generic and disease-specific quality of life as a predictor of long-term mortality in heart failure. Eur J Heart Fail 2010, 12:1372-1378.

5. Grool AM, van der Graaf Y, Visseren FL, de Borst GJ, Algra A, Geerlings MI, SMART Study Group: Self-rated health status as a risk factor for future vascular events and mortality in patients with symptomatic and asymptomatic atherosclerotic disease: the SMART study. J Intern Med 2012, 272:277-286.

6. Sehlen S, Marten-Mittag B, Herschbach P, Schweden M, Book K, Henrich G, Dühmke $E$, Dinkel A: Health-related quality of life supersedes other psychosocial predictors of long-term survival in cancer patients undergoing radiotherapy. Acta Oncol 2012, 51:1020-1028.

7. Michaud K, Vera-Llonch M, Oster G: Mortality risk by functional status and health-related quality of life in patients with rheumatoid arthritis. J Rheumatol 2012, 39:54-59.

8. Kanwal F, Gralnek IM, Hays RD, Zeringue A, Durazo F, Han SB, Saab S, Bolus R, Spiegel BM: Health-related quality of life predicts mortality in patients with advanced chronic liver disease. Clin Gastroenterol Hepatol 2009, 7:793-799.

9. Hayashino Y, Fukuhara S, Akiba T, Akizawa T, Asano Y, Saito S, Kurokawa K: Low health-related quality of life is associated with all-cause mortality in patients with diabetes on haemodialysis: the Japan Dialysis Outcomes and Practice Pattern Study. Diabet Med 2009, 26:921-927.

10. Osthus TB, von der Lippe N, Ribu L, Rustøen T, Leivestad T, Dammen T, Os I: Health-related quality of life and all-cause mortality in patients with diabetes on dialysis. BMC Nephrol 2012, 13:78

11. Haring R, Feng YS, Moock J, Völzke H, Dörr M, Nauck M, Wallaschofski H, Kohlmann T: Self-perceived quality of life predicts mortality risk better than a multi-biomarker panel, but the combination of both does best. BMC Med Res Methodol 2011, 11:103.

12. Ware J Jr, Kosinski M, Keller SD: A 12-item short-form health survey: construction of scales and preliminary tests of reliability and validity. Med Care 1996, 34:220-233.

13. Burstrom B, Fredlund P: Self rated health: is it as good a predictor of subsequent mortality among adults in lower as well as in higher social classes? J Epidemiol Community Health 2001, 55:836-840.

14. Wee CC, Davis RB, Hamel MB: Comparing the SF-12 and SF-36 health status questionnaires in patients with and without obesity. Health Qual Life Outcomes 2008, 6:11.

15. Ul-Haq Z, Mackay DF, Martin D, Smith DJ, Gill JM, Nicholl Bl, Cullen B, Evans J, Roberts B, Deary IJ, Gallacher J, Hotopf M, Craddock N, Pell JP: Heaviness, health and happiness: a cross-sectional study of 163066 UK Biobank participants. J Epidemiol Community Health 2014, 68:340-348

16. Ul-Haq Z, Mackay D, Fenwick E: Association between self-reported general and mental health and adverse outcomes; a retrospective cohort study of 19625 Scottish adults. PLoS One 2014, 9:e93857.

17. Forero CG, Vilagut G, Adroher ND, Alonso J: Multidimensional item response theory models yielded good fit and reliable scores for the Short Form-12 questionnaire. J Clin Epidemiol 2013, 66:790-801.

18. Delpierre C, Kelly-Irving M, Munch-Petersen M, Lauwers-Cances V, Datta GD, Lepage B, Lang T: SRH and HrQOL: does social position impact differently on their link with health status? BMC Public Health 2012, 12:19.

19. Bowling A: Just one question: if one question works, why ask several? J Epidemiol Community Health 2005, 59:342-345.

20. UI-Haq Z, Mackay DF, Fenwick E, Pell JP: Impact of metabolic comorbidity on the association between body mass index and health-related quality of life: a Scotland-wide cross-sectional study of 5,608 participants. BMC Public Health 2012, 12:143.

21. UI-Haq Z, Mackay DF, Fenwick E, Pell JP: Meta-analysis of the association between body mass index and health-related quality of life among children and adolescents, assessed using the pediatric quality of life inventory index. J Pediatr 2013, 162:280-286.

22. UI-Haq Z, Mackay DF, Fenwick E, Pell JP: Meta-analysis of the association between body mass index and health-related quality of life among adults, assessed by the SF-36. Obesity 2013, 21:E322-E327.

23. Duncan M, Griffith M, Rutter H, Goldacre MJ: Certification of obesity as a cause of death in England 1979-2006. Eur J Public Health 2010, 20:671-675
24. Guh DP, Zhang W, Bansback N, Amarsi Z, Birmingham CL, Anis AH: The incidence of co-morbidities related to obesity and overweight: a systematic review and meta-analysis. BMC Public Health 2009, 9:88.

25. UI-Haq Z, Mackay DF, Fenwick E, Pell JP: Association between body mass index and mental health among Scottish adult population: a cross-sectional study of 37272 participants. Psychol Med 2013, 44:1-10.

26. Gray L, Batty GD, Craig P, Stewart C, Whyte B, Finlayson A, Leyland AH: Cohort profile: the Scottish health surveys cohort: linkage of study participants to routinely collected records for mortality, hospital discharge, cancer and offspring birth characteristics in three nationwide studies. Int J Epidemiol 2010, 39:345-350.

27. NHS Scotland: Hospital Records Data Monitoring. http://www.isdscotland. org/Products-and-Services/Hospital-Records-Data-Monitoring/SMRCompleteness/ 2010 (accessed 06 Mar 2014)

28. Tsai SY, Chi LY, Lee CH, Chou P: Health-related quality of life as a predictor of mortality among community-dwelling older persons. Eur J Epidemiol 2007, 22:19-26.

29. Dorr DA, Jones SS, Burns L, Donnelly SM, Brunker CP, Wilcox A, Clayton PD: Use of health-related, quality-of-life metrics to predict mortality and hospitalizations in community-dwelling seniors. J Am Geriatr Soc 2006, 54:667-673

30. Otero-Rodriguez A, Leon-Munoz LM, Balboa-Castillo T, Banegas JR, Rodríguez-Artalejo F, Guallar-Castillón P: Change in health-related quality of life as a predictor of mortality in the older adults. Qual Life Res 2010, 19:15-23.

31. Nielsen AB, Siersma $V$, Hiort LC, Drivsholm $T$, Kreiner $S$, Hollnagel $H$ : Self-rated general health among 40-year-old Danes and its association with all-cause mortality at 10-, 20-, and 29 years' follow-up. Scand J Public Health 2008, 36:3-11

32. Idler EL, Russell LB, Davis D: Survival, functional limitations, and self-rated health in the NHANES I Epidemiologic Follow-up Study, 1992. First National Health and Nutrition Examination Survey. Am J Epidemiol 2000, 152:874-883.

33. Idler EL, Benyamini Y: Self-rated health and mortality: a review of twenty-seven community studies. J Health Soc Behav 1997, 38:21-37.

34. Kenchaiah S, Evans JC, Levy D, Wilson PW, Benjamin EJ, Larson MG, Kannel WB, Vasan RS: Obesity and the risk of heart failure. $N$ Engl J Med 2002, 347:305-313

35. Eckel $\mathrm{RH}$ : Obesity and heart disease: a statement for healthcare professionals from the Nutrition Committee, American Heart Association. Circulation 1997, 96:3248-3250.

36. Bianchini F, Kaaks R, Vainio H: Overweight, obesity, and cancer risk. Lancet Oncol 2002, 3:565-574.

37. Flegal KM, Kit BK, Orpana H, Graubard BI: Association of all-cause mortality with overweight and obesity using standard body mass index categories: a systematic review and meta-analysis. JAMA 2013, 309:71-82.

38. Janssen I, Mark AE: Elevated body mass index and mortality risk in the elderly. Obes Rev 2007, 8:41-59.

39. McGee DL: Body mass index and mortality: a meta-analysis based on person-level data from twenty-six observational studies. Ann Epidemiol 2005, 15:87-97.

40. Saquib N, Brunner R, Kubo J, Tindle H, Kroenke C, Desai M, Daviglus ML, Allen N, Martin LW, Robinson J, Stefanick ML: Self-perceived physical health predicts cardiovascular disease incidence and death among postmenopausal women. BMC Public Health 2013, 13:468.

41. Forero CG, Adroher ND, Stewart-Brown S, Castellvi P, Codony M, Vilagut G, Mompart A, Tresseres R, Colom J, Castro Jl, Alonso J: Differential item and test functioning methodology indicated that item response bias was not a substantial cause of country differences in mental well-being. J Clin Epidemiol 2014, doi:10.1016/j.jclinepi.2014.06.017.

\section{doi:10.1186/1471-2458-14-1197}

Cite this article as: Ul-Haq et al:: Association between physical and mental health-related quality of life and adverse outcomes; a retrospective cohort study of 5,272 Scottish adults. BMC Public Health 2014 14:1197. 